\title{
Wide Area Protection Scheme Preventing Cascading Events Caused by Load Flow Transferring
}

\author{
Zhou Liu, Zhe Chen \\ Department of Energy Technology \\ Aalborg University \\ Aalborg, Denmark \\ zli@et.aau.dk, zch@et.aau.dk
}

\author{
Haishun Sun \\ Huazhong University of Science and \\ Technology \\ Wuhan, China \\ haishunsun@hust.edu.cn
}

\author{
Yanting $\mathrm{Hu}$ \\ School of Engineering Education \\ Glyndwr University \\ Wrexham, UK \\ y.hu@glyndwr.ac.uk
}

\begin{abstract}
Load flow transferring after an initial contingency is regarded as one of the main reasons of causing unexpected cascading trips. A multi agent system (MAS) based wide area protection strategy is proposed in this paper to predict the load flow transferring from the point of view of impedance relays, and prevent the unexpected relay operations accordingly. The variations of node injections during the post fault transient period will be also considered in the prediction algorithm. The power system of Eastern Denmark modeled in real time digital simulator (RTDS) will be used to demonstrate the proposed strategy. The simulation results indicate this strategy can successfully predict and prevent the unexpected relay operation caused by load flow transferring.
\end{abstract}

Index Terms-Cascading trips, load flow transferring, multi agent system, wide area protection

\section{INTRODUCTION}

Nowadays, power system blackouts are regarded as the most severe disasters which could result in devastating effects on human society and industry. Especially with the modern development, power system is operated nearer to operation margin and with less redundancy [1], which is already in a high risk of overloading operation situation. Also, recent large scale blackouts in USA and Europe [2] [3] give an alarm on the vulnerability of prevailing power systems.

When N-1 (or N-k) contingency occurs in a heavily loaded power system, a large amount of load flow will be re distributed and transferred to the remained transmission network, which may cause those critical transmission lines overloaded. Then the related backup relays may mistakenly take the overload situation as a remote fault to trip the some transmission lines unexpectedly, which will overload the rest system further. Consequently, cascading trips and network splitting may happen inevitably. However, if the load flow transferring can be identified timely and the trip of the critical overload lines is delayed/blocked quickly, the overload situation might be mitigated by timely emergent protection and control schemes.

So far, many research efforts have been taken to prevent cascading events. One emergent prediction control algorithm is based on dc load flow sensitivities, including line outage distribution factor (LODF) and generator shift factor (GSF). The method predicts the component outage caused by load flow transferring and distinguishes the overload situation [4] [5]. Moreover, the variation of system state variables during transient period after an outage has also been studied over the whole system [6] or by local measured information [7]. However, those dc load flow based predicting methods only consider the flow of active power and assume bus voltages and reactive power flow being constant, which is not the case in real situation, especially in the cascading events.

In this paper, with the aim of preventing the unexpected relay operation caused by load flow transferring in the post fault stage, a sensitivity method based on impedance principle is presented to predict and identify the possible overloaded line caused by load flow transferring in the point of view of relays, the variations of both active power and reactive power are considered. Furthermore, in order to eliminate the unexpected relay operation which will normally have a negative effect on system stability, MAS will be applied for wide area information exchanging and sharing. The power system of eastern demark will be used as test system and modeled in RTDS to demonstrate the proposed prediction algorithm and protection strategy. The rest of paper is organized as follows: a brief introduction of the sensitivity based prediction and identification algorithm is presented in Section II; multi agent system based control strategy is described in Section III; then in Section IV, the strategy is demonstrated on the power system modeled in RTDS; and finally, the conclusion is made in Section V.

\section{PREDICTION AMD IDENFICATION ALGORITHM}

\section{A. Relay operation characteristics}

In this paper, the impedance relay is used as an example and the zone 3 relay will be the studied objective. The classic characteristics of impedance relay on the line $i j$ are shown in Figure 1. $V_{i} \angle \theta_{i}, V_{j} \angle \theta_{j}$ represent the bus voltages of the line $i j$ ends respectively. $Z_{i j} \angle \varphi_{\text {lineij }}$ is the impedance of line $i j$. $Z_{\text {Tset }}, Z_{\text {Tset } i j}$ are the zone 3 set values at the impedance angle $\varphi_{\text {lineij }}$ and power factor angle $\varphi_{i j}$ respectively. $Z_{a i j} \angle \varphi_{i j}$ is the measured impedance by the relay. At current power factor angle $\varphi_{i j}$, the relay operation margin can be expressed as: 
where

$$
M_{T i j}=Z_{a i j}-Z_{T s e t i j}
$$

$$
\begin{gathered}
Z_{a i j}=\frac{Z_{i j} V_{i}}{\sqrt{\left(V_{i}-V_{j} \cos \theta_{i j}\right)^{2}+\left(V_{j} \sin \theta_{i j}\right)^{2}}} \\
Z_{\text {Tsetij }}=Z_{\text {Tset }} \cos (\Delta \varphi) \\
\Delta \varphi=\varphi_{\text {lineij }}-\varphi_{i j}
\end{gathered}
$$

The set value of zone 3 relay is defined according to NERC proposed relay loadability limit [8], which is given by:

$$
Z_{\text {Tset }}=\frac{V_{\text {min }}^{2}}{S_{\text {max }} \cos \left(\varphi_{\text {line }}-\varphi_{\max }\right)}
$$

where $S_{\max }, V_{\min }, \varphi_{\text {line }}, \varphi_{\max }$ represent the load capacity limit of the line, low voltage limit of the bus, impedance angle of the line and maximum load flow angle respectively.

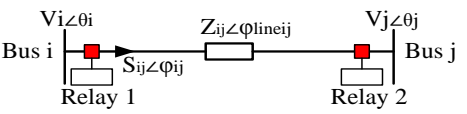

(a) Transmission line with impedance relays

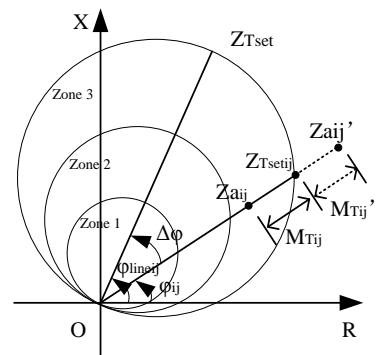

(b) Characteristics of three zone impedance relay

Figure 1. Traditional impedance relay on transmission line

Normally, the measured impedance $Z_{a i j}^{\prime}$ should be located outside the zone 3 circle area and the relay operation margin is positive as expressed by $M_{T i j}^{\prime}$ in Fig. 1 . When $Z_{a i j}$ enters the zone 3 area, the backup relay will be initiated to trip the line within a preset delay. At this situation, the sign of $M_{T i j}$ is changed to be negative. If this negative margin is caused by the post fault load flow transferring, this trip will be regarded as unexpected one because it is not functioned to clear a fault in its backup relay zone, and would induce overloaded on other lines and might further result in cascaded events.

\section{B. Impedance sensitivity to system operation variables}

With the aim of finding the influence factors between relay measured impedance and load flow transferring, the linearization method can be applied here. The impedance seen by Relay 1 in Fig. 1 can be rewritten with (6) (7) (8).

$$
\begin{gathered}
\overline{Z_{a i j}}=R_{a i j}+j X_{a i j} \\
R_{a i j}=\frac{V_{i}\left(G_{i j} V_{j} \cos \theta_{i j}+B_{i j} V_{j} \sin \theta_{i j}-G_{i j} V_{i}\right)}{\left(B_{i j}^{2}+G_{i j}^{2}\right)\left(V_{i}^{2}+V_{j}^{2}-2 V_{i} V_{j} \cos \theta_{i j}\right)} \\
X_{a i j}=\frac{V_{i}\left(B_{i j} V_{i}+G_{i j} V_{j} \sin \theta_{i j}-B_{i j} V_{j} \cos \theta_{i j}\right)}{\left(B_{i j}^{2}+G_{i j}^{2}\right)\left(V_{i}^{2}+V_{j}^{2}-2 V_{i} V_{j} \cos \theta_{i j}\right)}
\end{gathered}
$$

where $\overline{Y_{i j}}={\overline{Z_{i j}}}^{-1}=G_{i j}+j B_{i j}$, then the linearized form of (7) (8) is given by (9) (10).

$$
\begin{aligned}
& \Delta R_{a i j}=C_{R \theta i} \Delta \theta_{i}+C_{R \theta j} \Delta \theta_{j}+C_{R v i} \Delta V_{i}+C_{R v j} \Delta V_{j} \\
& \Delta X_{a i j}=C_{X \theta i} \Delta \theta_{i}+C_{X \theta j} \Delta \theta_{j}+C_{X v i} \Delta V_{i}+C_{X v j} \Delta V_{j}
\end{aligned}
$$

where $C_{i j}$ is the sensitivity matrix including the sensitivities of impedance $\left(R_{a i j}, X_{a i j}\right)$ to related bus voltages $\left(\theta_{i}, \theta_{j}, V_{i}, V_{j}\right), C_{R i j}$ and $C_{X i j}$ are submatrices of $C_{i j}$.

$$
\begin{aligned}
C_{i j} & =\left[\begin{array}{l}
C_{R i j} \\
C_{X i j}
\end{array}\right]=\left[\begin{array}{llll}
C_{R \theta i} & C_{R \theta j} & C_{R v i} & C_{R v j} \\
C_{X \theta i} & C_{X \theta j} & C_{X v i} & C_{X v j}
\end{array}\right] \\
& =\left[\begin{array}{llll}
\frac{\partial R_{a i j}}{\partial \theta_{i}} & \frac{\partial R_{a i j}}{\partial \theta_{j}} & \frac{\partial R_{a i j}}{\partial V_{i}} & \frac{\partial R_{a i j}}{\partial V_{j}} \\
\frac{\partial X_{a i j}}{\partial \theta_{i}} & \frac{\partial X_{a i j}}{\partial \theta_{j}} & \frac{\partial X_{a i j}}{\partial V_{i}} & \frac{\partial X_{a i j}}{\partial V_{j}}
\end{array}\right]
\end{aligned}
$$

Taking all the concerned relays into consideration, the sensitivity of impedances can be expressed in the matrix form as (12); also based on load flow Jacobin $\boldsymbol{J}$ defined by (15), the sensitivity $\boldsymbol{H}$ of impedance to node powers can be derived from (13), where $\boldsymbol{C}_{\boldsymbol{R} \boldsymbol{\theta}}, \boldsymbol{C}_{\boldsymbol{R} \boldsymbol{V}}, \boldsymbol{C}_{\boldsymbol{X} \boldsymbol{\theta}}$ and $\boldsymbol{C}_{\boldsymbol{X} \boldsymbol{V}}$ are submatrices of $\boldsymbol{C}$; $\boldsymbol{H}_{R P}, \boldsymbol{H}_{R Q}, \boldsymbol{H}_{X P}$ and $\boldsymbol{H}_{X Q}$ are submatrices of $\boldsymbol{H}$.

$$
\begin{aligned}
& {\left[\begin{array}{l}
\Delta R_{a} \\
\Delta X_{a}
\end{array}\right]=C\left[\begin{array}{l}
\Delta \theta \\
\Delta V
\end{array}\right]=\left[\begin{array}{ll}
C_{R \theta} & C_{R V} \\
C_{X \theta} & C_{X V}
\end{array}\right]\left[\begin{array}{l}
\Delta \theta \\
\Delta V
\end{array}\right]} \\
& {\left[\begin{array}{l}
\Delta R_{a} \\
\Delta X_{a}
\end{array}\right]=H\left[\begin{array}{l}
\Delta P \\
\Delta Q
\end{array}\right]=\left[\begin{array}{ll}
H_{R P} & H_{R Q} \\
H_{X P} & H_{X Q}
\end{array}\right]\left[\begin{array}{l}
\Delta P \\
\Delta Q
\end{array}\right]}
\end{aligned}
$$

where

$$
\begin{gathered}
H=C J^{-1} \\
{\left[\begin{array}{c}
\Delta P \\
\Delta Q
\end{array}\right]=J\left[\begin{array}{l}
\Delta \theta \\
\Delta V
\end{array}\right]=\left[\begin{array}{ll}
J_{P \theta} & J_{P V} \\
J_{Q \theta} & J_{Q V}
\end{array}\right]\left[\begin{array}{l}
\Delta \theta \\
\Delta V
\end{array}\right]=\left[\begin{array}{ll}
\frac{\partial \Delta P}{\partial \theta} & \frac{\partial \Delta P}{\partial V} \\
\frac{\partial \Delta Q}{\partial \theta} & \frac{\partial \Delta Q}{\partial V}
\end{array}\right]\left[\begin{array}{c}
\Delta \theta \\
\Delta V
\end{array}\right]}
\end{gathered}
$$

\section{Flow transferring prediction and identification}

In order to predict the post fault load flow transferring based on pre fault system data, the simulation method of line outage is shown in Figure 2. Figure 2 (a) is a network operated in normal situation without line outage. Suppose line $l$ from bus $m$ to bus $n$ were opened by circuit breakers due to some kind of faults, as shown in Figure 2 (b). A line outage may be modeled by adding two virtual power injections $\left(\Delta P_{m}, \Delta Q_{m}, \Delta P_{n}, \Delta Q_{n}\right)$ to the network, one at each end of the line to be dropped [9], which is shown in Figure 2 (c). At this situation of Figure 2 (c), the line is still kept in the network, and the effects of its being dropped are modeled by the virtual injections.

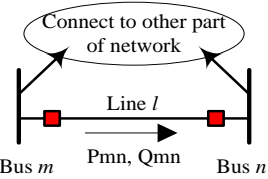

(a) Network before line $l$ outage

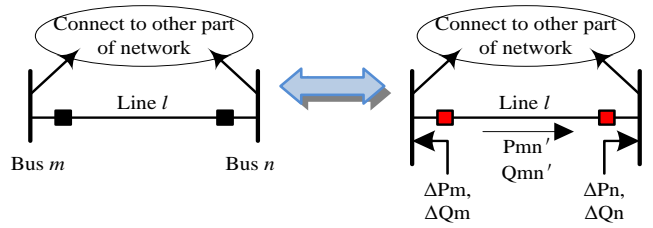

(b) Network after line l outage (c) Modeling the outage by injections

Figure 2. Line outage simulation in power network 
With the aim of making these two situations have the equal effect, the necessary requirements should be satisfied as:

$$
\begin{gathered}
P_{m n}^{\prime}=P_{m n}+\Delta P_{m n}=\Delta P_{m}=-\Delta P_{n} \\
Q_{m n}^{\prime}=Q_{m n}+\Delta Q_{m n}=\Delta Q_{m}+\frac{b}{2} V_{m}^{\prime 2} \\
\quad=-\Delta Q_{n}+\frac{P_{m n}^{\prime 2}+Q_{m n}^{\prime 2}}{V_{m}^{\prime 2}} X_{m n}-\frac{b}{2} V_{n}^{\prime 2}
\end{gathered}
$$

where

$$
\begin{aligned}
& V_{m}^{\prime}=V_{m}+\Delta V_{m} \\
& V_{n}^{\prime}=V_{n}+\Delta V_{n}
\end{aligned}
$$

$P_{m n}, Q_{m n}, V_{m}, V_{n}$ represent the line flows and node voltages in the pre fault state; $P_{m n}^{\prime}, Q_{m n}^{\prime}, V_{m}^{\prime}, V_{n}^{\prime}$ represent these variables in the post fault state; $\Delta P_{m n}, \Delta Q_{m n}, \Delta V_{m}$ and $\Delta V_{n}$ represent the variations of these variables when the virtual injections have been applied, $b$ is the susceptance of line $l$ to ground.

In this way, it can be assumed that line $l$ is isolated from the remainder of the network even though the breakers are closed. Compared the situation in Figure 2 (c) with Figure 2 (a), the variations are mainly expressed by those virtual injections. So if the $\Delta P_{m}, \Delta Q_{m}$ and $\Delta P_{n}, \Delta Q_{n}$ can be obtained, the variation of impedances in the relays of rest lines can be easily deduced by (13) based on pre fault system data.

\section{1) Virtual injection powers calculation}

The line flows $P_{m n}, Q_{m n}$ of the line $l$ can be expressed as:

$$
\begin{aligned}
& P_{m n}=V_{m} V_{n}\left(G_{m n} \cos \theta_{m n}+B_{m n} \sin \theta_{m n}\right)-G_{m n} V_{m}^{2} \\
& Q_{m n}=V_{m} V_{n}\left(G_{m n} \sin \theta_{m n}-B_{m n} \cos \theta_{m n}\right)+B_{m n} V_{m}^{2}
\end{aligned}
$$

Then the linear relationship between line flow and these virtual injection powers can be expressed as:

$$
\begin{gathered}
{\left[\begin{array}{c}
\Delta P_{m n} \\
\Delta Q_{m n}
\end{array}\right]=D_{m n}\left[\begin{array}{c}
\Delta \theta_{m} \\
\Delta \theta_{n} \\
\Delta V_{m} \\
\Delta V_{n}
\end{array}\right]=L_{m n}\left[\begin{array}{c}
\Delta P_{m} \\
\Delta P_{n} \\
\Delta Q_{m} \\
\Delta Q_{n}
\end{array}\right]} \\
L_{m n}=D_{m n} J_{m n m n}
\end{gathered}
$$

where $D_{m n}$ is the sensitivity matrix including the sensitivities of line flow to the bus voltages $\left(\theta_{i}, \theta_{j}, V_{i}, V_{j}\right)$, $L_{m n}$ is the sensitivity matrix of line flow to virtual injections. $J_{m n m n}$ is the submatrix of load flow Jacobin $J$.

According to load flow sensitivities in the pre fault state, $\Delta \mathrm{V}_{\mathrm{m}}$ and $\Delta V_{n}$ can be also expressed by $\Delta P_{m}, \Delta Q_{m}, \Delta P_{n}, \Delta Q_{n}$, then based on equations (16)-(23), $\Delta P_{m}, \Delta Q_{m}, \Delta P_{n}, \Delta Q_{n}$ can be calculated easily.

\section{2) Sensitivity based relay impedacne prediction}

When the virtual injection variations $\Delta P_{m}, \Delta Q_{m}$ and $\Delta P_{n}, \Delta Q_{n}$ are obtained, the impedance seen by relays in the post fault state can be previously calculated based on the pre fault sensitivities. Based on equation (13), the predicted impedance variations $\left(\Delta R_{a i j}^{P l}, \Delta X_{a i j}^{P l}\right)$ on any transmission line $i j$, after line $l$ is tripped, can be calculated by:

$$
\left[\begin{array}{c}
\Delta R_{a i j}^{P l} \\
\Delta X_{a i j}^{P l}
\end{array}\right]=C_{i j} J_{i j m n}{ }^{-1}\left[\begin{array}{c}
\Delta P_{m} \\
\Delta P_{n} \\
\Delta Q_{m} \\
\Delta Q_{n}
\end{array}\right]=H_{i j m n}\left[\begin{array}{c}
\Delta P_{m} \\
\Delta P_{n} \\
\Delta Q_{m} \\
\Delta Q_{n}
\end{array}\right]
$$

where $J_{i j m n}$ is the submatrix of load flow Jacobin $\boldsymbol{J}$, and $H_{i j m n}$ is the submatrix of $\boldsymbol{H}$ defined by (14).

\section{3) Flow transferring identification}

After the line $l$ is tripped by the related relay to clear the fault, the practical impedance can be real time measured by relays on the key transmission lines, named by $Z_{a i j}^{M}$; and the predicted one can be named by $Z_{a i j}^{P}$. It can be inferred that in those load flow transferring cases, $Z_{a i j}^{P}$ and $Z_{a i j}^{M}$ will be approximately same; while in the cases of internal faults, this condition can not be satisfied. In order to distinguish the load flow transferring from internal faults, the identification criterion can be written as:

$$
\left|Z_{a i j}^{M}-Z_{a i j}^{P}\right|<\varepsilon\left|Z_{a i j}^{P}\right|
$$

where

$$
\begin{aligned}
& Z_{a i j}^{M}=R_{a i j}^{M}+j X_{a i j}^{M} \\
& Z_{a i j}^{P}=R_{a i j}^{P}+j X_{a i j}^{P} \\
& =R_{a i j}^{0}+\Delta R_{a i j}^{P}+\mathrm{j}\left(X_{a i j}^{0}+\Delta X_{a i j}^{P}\right)
\end{aligned}
$$

$R_{a i j}^{0}$ and $X_{a i j}^{0}$ are measured impedance in the pre fault state; $\Delta R_{a i j}^{P}$ and $\Delta X_{a i j}^{P}$ are predicted variation of the impedance in the post fault state; $\varepsilon$ represents the threshold of error caused by linearization and neglecting transient characteristics of components. If the criterion holds, this relay will define this trigger caused by flow transferring. It should be blocked or extend the delay settings until this line reached its thermal limit, and the emergent countermeasures should be taken to eliminate the overload situation. Otherwise, if the criterion cannot hold, the relay will define this situation as an internal fault and trip the line according to traditional functions.

\section{Transient period consideration}

So far, the network structure and all other node injections have been assumed unchanged. However, during transient period in the post fault stage, node injection powers are not constant, especially the reactive powers of generators, which is the main reason of error production. Assuming the load model in this paper applies static constant power load, the variation of generator node injection should be considered in the impedance prediction algorithm. Consequently, according to superposition principle, the final predicted relay impedance variation $\left(\Delta R_{a i j}^{P}, \Delta X_{a i j}^{P}\right)$ on line $i j$, after line $l$ is tripped, can be expressed as:

$$
\left[\begin{array}{c}
\Delta R_{a i j}^{P} \\
\Delta X_{a i j}^{P}
\end{array}\right]=\left[\begin{array}{c}
\Delta R_{a i j}^{P l} \\
\Delta X_{a i j}^{P l}
\end{array}\right]+\left[\begin{array}{c}
\Delta R_{a i j}^{P g} \\
\Delta X_{a i j}^{P g}
\end{array}\right]
$$

where

$$
\left[\begin{array}{c}
\Delta R_{a i j}^{P g} \\
\Delta X_{a i j}^{P g}
\end{array}\right]=C_{i j} J_{i j g}{ }^{-1}\left[\begin{array}{c}
\Delta P_{g 1} \\
\vdots \\
\Delta P_{g n} \\
\Delta Q_{g 1} \\
\vdots \\
\Delta Q_{g n}
\end{array}\right]=H_{i j g}\left[\begin{array}{c}
\Delta P_{g 1} \\
\vdots \\
\Delta P_{g n} \\
\Delta Q_{g 1} \\
\vdots \\
\Delta Q_{g n}
\end{array}\right]
$$

$J_{i j g}$ and $H_{i j g}$ are the submatrices of $\boldsymbol{J}$ and $\boldsymbol{H}$ respectively, which represent the relationship of node voltages and relay impedances to generator node injections. $\Delta P_{g 1}, \Delta Q_{g 1} \cdots \Delta P_{g n}$, $\Delta Q_{g n}$ represent the variations of $n$ generator node injections.

Actually, the sensitivity matrix $\boldsymbol{H}$ is a high order matrix and the values of most elements in each line are quite small, 
which means for specific branch in the network, few node injections have the significant influence on the impedance. So in order to decrease the measurement points in the post fault stage, the generator nodes with bigger sensitivities will be chosen to be considered in the prediction algorithm.

\section{STRATEGY IMPLEMENTATION}

In order to implement the proposed wide area protection against cascading trips caused by load flow transferring, multi agent system based control strategy will be adopted, as shown in Figure 3.

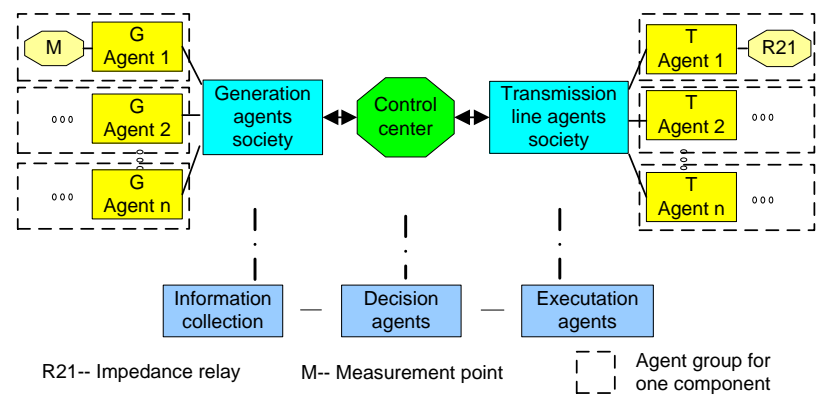

Figure 3. The structure of MAS based control system

This MAS based control system adopts a three-level hierarchical control structure, which is comprised by distributed agent level, cooperation society level and central processing level. Moreover, every agent in this system has three basic functions: information collection, decision making according to the prevailing state and decision execution [10].

In the distributed agent level, relay agents on transmission lines will monitor and send the states of breaker, relay and line flows to control center (CC). When the critical relay is triggered, the normal functions will perform as usual, namely, this relay will trip if the time delay is reached. As for zone 3 relay, the delay time is typical $500 \mathrm{~ms}$ or longer, so there is enough time for relay agent to communicate with CC and obtain the information about line outage and variations of node injections (including virtual ones). Then the predicted post fault impedance $Z_{a i j}^{P}$ can be calculated timely. Based on the criterion (25), whether this relay is triggered by load flow transferring can be identified. If the criterion holds, this agent will block the tripping signal to avoid the probable cascading trips. Meanwhile, an alarm signal will be sent to CC for emergent control execution. Besides, the agents on generator nodes will monitor the states of generators and supply the information about injection variations to CC and relay agents.

The distributed agents in the lowest level can be grouped into agent societies according to the similarity of their functions, such as generation agent society, transmission agent society, etc. This is the middle level which builds an effective cooperation environment between agents. A control center (CC) agent is the highest level of the MAS and is designed to coordinate with all the lower level agents executing wide area protection strategy. Based on the data received from distributed agents in the whole system, the topology of network and related sensitivity matrices can be produced accordingly. When $\mathrm{CC}$ detect the network changes, the related sensitivities and node injection variations will be sent to distributed relay agents for their local prediction and identification. When CC receive the information about relay blocking and alarm signals, emergent control strategies will be applied to adjust system to normal state (which will be discussed in another paper). Then CC will calculate new sensitivities according to current network topology.

\section{CASE STUDY}

The test system model which is simplified from eastern Denmark is built in RSCAD/RTDS with two racks and depicted in Runtime/RTDS, as shown in Figure 4. There are 19 buses with voltages from $0.7 \mathrm{kV}$ to $400 \mathrm{kV}$, four central power plants and their control (G01, G11, G21, G22), a Static VAR compensator (SVC), several consumption centers modeled by constant power load, a lumped equivalent of local wind turbines and an equivalent of a large offshore wide farm [11]. The wind turbines are fixed-speed, equipped with conventional asynchronous generators, which is similar to the situation of Danish wind farm in 2003. About $70 \%$ of total load consumption is located at bus 12 .

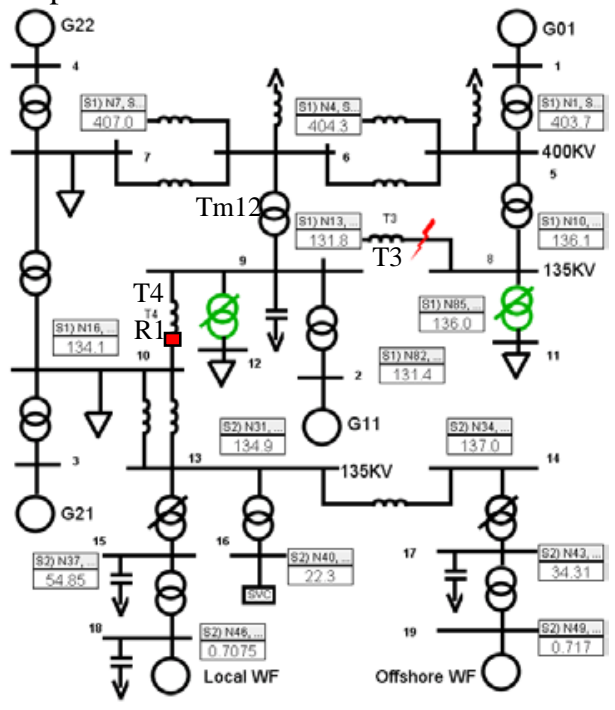

Figure 4. Test system model in Runtime/RTDS

Suppose at 3s, a three phase permanent short circuit fault occurred on T3, then T3 was tripped 0.1 s later. The zone 3 relay on $\mathrm{T} 4$ and $\mathrm{Tm} 12$ was triggered due to load flow transferring, the relay R1 on T4 is chosen as a studied example, the line flow and impedance seen by R1 are shown in Figure 5.

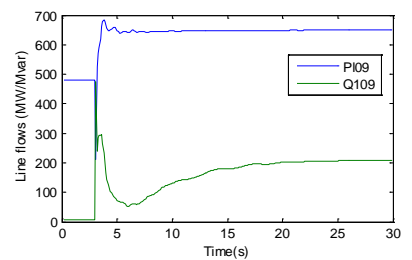

(a) Line flow

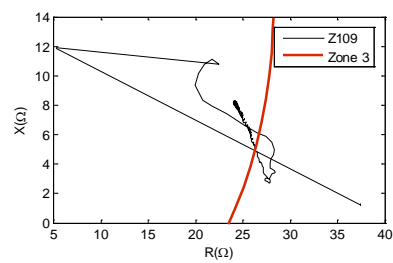

(b) Impedance locus of relay R1
Figure 5. Line flow and impedance seen by R1 on line 109

When the proposed protection strategy is taken into effect, the virtual injections and the sensitivities based on pre fault system data can be obtained, as shown in Table I. 
TABLE I.

VIRTUAL INJECTIONS AND RELATED SENSITIVITY

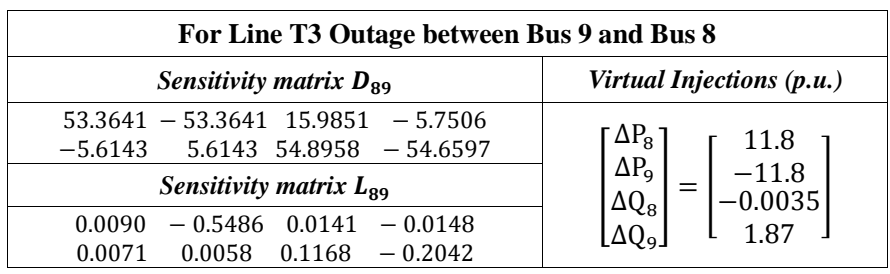

Then based on the impedance sensitivity method described in Section II, the related sensitivities and the predicted impedance variation can be obtained, as shown in Table II.

TABLE II. IMPEDANCE PREDICTION ON T4

\begin{tabular}{|c|c|}
\hline \multicolumn{2}{|c|}{ For Line T4 between Bus 10 and Bus 9} \\
\hline Sensitivity matrix $C_{109}$ & Predicted impedance (ohm) \\
\hline $\begin{array}{rrrr}-1.1346 & 1.1346 & -0.0312 & 0.0318 \\
-0.0310 & 0.0310 & 1.1422 & -1.1621 \\
\end{array}$ & {$\left[\begin{array}{c}R_{a 109}^{0} \\
v^{0}\end{array}\right]=\left[\begin{array}{c}37.45 \\
125\end{array}\right]$} \\
\hline Sensitivity matrix $H_{10989}$ & $\begin{array}{l}\left.X_{a 109}\right\lrcorner[1.25] \\
P l\end{array}$ \\
\hline $\begin{array}{cccc}0.0001 & 0.0064 & 0.0006 & 0.0005 \\
-0.000 & -0.0009 & -0.0007 & -0.0057\end{array}$ & {$\left[\begin{array}{l}\Delta R_{a 109}^{P l} \\
\Delta X_{a 109}^{P l}\end{array}\right]=\left[\begin{array}{c}-13.3589 \\
0.0365\end{array}\right]$} \\
\hline
\end{tabular}

With consideration of node injection variation, if the delay communication takes 5 circles, the predicting calculation can be started after 10 circles, namely $0.2 \mathrm{~s}$ after the fault. Here the nodes of generator G01, G11, G21 and G22 will be chosen to be monitored. Then the injection variation and predicted curves can be shown in Figure 6.

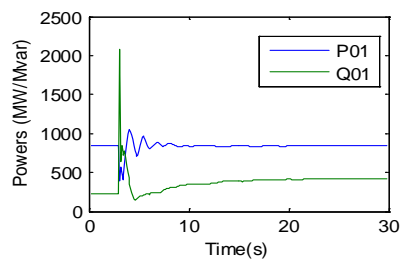

(a) Power injection from G01

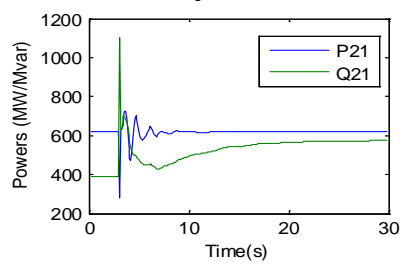

(c) Power injection from G21

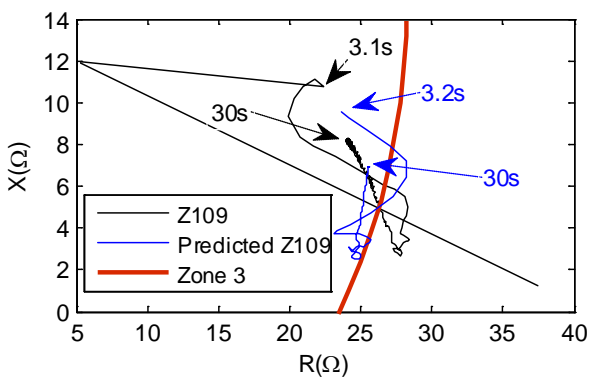

(e) Predicted impedance locus of relay R1

Figure 6. Generator injection variation and predicted impedance locous
From Figure 6 (e), the biggest error about $3 \Omega$ between predicted impedance (blue locus) and measured impedance (black locus) appeared just after the fault was cleared. But in about $500 \mathrm{~ms}$, the error decreased into $2 \Omega$. So the threshold of identification criterion (25) can be set as $2 \Omega$, and $\varepsilon$ applys 0.08 . Then this load flow transferring induced relay operation can be prevented by the proposed protection strategy in Section III.

\section{CONCLUSTION}

In this paper, a multi agent system based protection strategy is proposed to prevent the post fault load flow transferring induced unexpected relay operation. Impedance sensitivity based prediction algorithm is used to predict the impedance locus in the post fault state, based on the pre fault system data. The injection variations of generator nodes during the post fault state, including both active and reactive powers, have been considered to correct the prediction algorithm. The RTDS based case study has demonstrated the proposed strategy can successfully predict the impedance reviewed by relay on key transmission lines and prevent the unexpected relay trips due to flow transferring in the post fault state.

\section{REFERENCES}

[1] P. Kundur, Power Sytem Stability and Control, McGraw-Hill Press, 1993

[2] T. Cutsem and C. Vournas, Voltage stability of Electric Power System, Springer, 2008.

[3] J. McCalley, O. Oluwaseyi, V. Krishnam, R. Dai, C. Singh, K. Jiang, "System Protection Schemes: Limitations, Risks, and Management," Final Report from PSERC, 2010.

[4] L. Cheng, B. Zhang, Z. Hao, J. Shu, Z. Bo, "Feasibility Study on Active Power Security Protection of Transmission Section", Power and Energy Engineering Conference, 2009, APPEEC, 2009.

[5] K. Yunus, G. Pinares, L. Tuan, L. Bertling, “A combined zone-3 relay blocking and sensitivity-based load shedding for voltage collapse prevention”, Innovative Smart Grid Technologies Conference Europe (ISGT Europe), 2010.

[6] T. Bi, H. Xu, S. Huang, Q. Yang, "Flow transferring identification algorithm with consideration of transient period", Power \& Energy Society General Meeting, 2009.

[7] X. Dong, L. Ding, K. Liu, S. Shi, B. Wang, L. Cui, Z. Bo, "Smart system protection based on local information", $10^{\text {th }}$ IET International Conference on Developments in Power System Protection (DPSP 2010), pp. 1-4, 2010.

[8] NERC, Aug. 2005, "Relay loadability exceptions: determination and application of practical relaying loadability ratings," [Online]. Available: http://www.nerc.com/ filez/spctf.html.

[9] J. Zhu, Optimization of Power System Operation, John Wiley \& Sons, Inc., Hoboken, New Jersey. 2009.

[10] Z. Liu, Z. Chen, H. Sun, C. Liu, "Control and Protection Cooperation Strategy for Voltage Instability", 47th International Universities' Power Engineering Conference (UPEC), 2012.

[11] V. Akhmatov, "Analysis of dynamic behaviour of electric power systems with large amount of wind power", Ph.D. dissertation, Dept. Electric Power. Eng., Technical Univ. Denmark, Kgs. Lyngby, 2003. 